\title{
Exploratory research session on the quantization of the gravitational field ${ }^{\star}$ At the Institute for Theoretical Physics, Copenhagen, Denmark, June-July 1957
}

\author{
Bryce S. DeWitt ${ }^{\mathrm{a}}$ \\ Aeronautical Research Laboratory, Contract No. AF 33(616)-5367, Wright Air \\ Development Center, Air Research and Development Command, United States \\ Air Force, Wright-Patterson Air Force Base, Dayton, Ohio, USA
}

\author{
Received 9 March 2017 / Accepted 15 March 2017 \\ Published online 1 June 2017 \\ (C) The Author(s) 2017. This article is published with open access \\ at Springerlink.com
}

\begin{abstract}
During the period June-July 1957 six physicists met at the Institute for Theoretical Physics of the University of Copenhagen in Denmark to work together on problems connected with the quantization of the gravitational field. A large part of the discussion was devoted to exposition of the individual work of the various participants, but a number of new results were also obtained. The topics investigated by these physicists are outlined in this report and may be grouped under the following main headings: The theory of measurement. Topographical ${ }^{1}$ problems in general relativity. Feynman quantization. Canonical quantization. Approximation methods. Special problems.
\end{abstract}

\section{Foreword}

An exploratory research session on problems connected with the quantization of the gravitational field was held from the 15th of June to the 15th of July 1957 at the

\footnotetext{
* Reproduced here from a copy of the original document available at the Aage Petersen Collection of Reprints and Manuscripts, Series I, Box 1, Folder 8, deposited at the Niels Bohr Library and Archives, American Institute of Physics, College Park, MD, United States. As far as the the editors know, that is the single remaining copy of the final, typed version of the document. The current version was edited and commented by Alexander Blum (Max-Planck-Institut für Wissenschaftsgeschichte, Berlin, e-mail: ablum@mpiwg-berlin.mpg.de) and Thiago Hartz (Museu de Astronomia e Ciências Afins, Rio de Janeiro, e-mail: thiagohartz@gmail.com). Their comments on the manuscript have been included in additional footnotes, marked as "Editors' comment". Thanks is due to Melanie J. Mueller, director of the Niels Bohr Library and Archives, for authorizing the reproduction of this document.

a deceased

1 Editors' comment: This is a typo. DeWitt means "topological".
} 
University Institute for Theoretical Physics, Copenhagen, Denmark. The following persons participated in this research session:

Professor Christian Møller, Director of CERN Theoretical Study Division, Institute for Theoretical Physics, Copenhagen, Denmark. ${ }^{2}$

Professor Oskar Klein, University of Stockholm, Stockholm, Sweden. ${ }^{2}$

Professor Bryce S. DeWitt, University of North Carolina, Chapel Hill, North Carolina.

Dr. Stanley Deser, Institute for Theoretical Physics, Copenhagen, Denmark.

Dr. Charles W. Misner, Princeton University, Princeton, New Jersey.

Dr. Bertel Laurent, University of Stockholm, Stockholm, Sweden. ${ }^{2}$

The session - or, rather, the sessions - were held in a very informal style on numerous mornings, afternoons, and evenings between the dates indicated. At the beginning there was some uncertainty as to how best to proceed. It was suggested, for example, that the group might attempt to tackle one specific problem with an eye toward eventual joint publication. It was soon realized, however, that this would be impractical, in view of the varied interests of the members of the group, the nature of the present outstanding problems in gravitational theory, and the fairly limited amount of time available. It was then proposed instead that each participant should endeavour to expound his own point of view and accomplishments to the others, and to proceed from there to further development, if possible. This, in the main, was the procedure adopted. It must be understood that the exposition itself was a lengthy process, involving many arguments at various temperature levels and frequent wandering into side issues. On the other hand, "plenary" sessions formed only a part of the activity. Often only two or three participants were involved in a single discussion, and much of the hard work was carried out in solitude.

The most noteworthy aspect of this mode of operation was a strong sense of luxury shared by the participants - luxury at having other experts close at hand, the luxury of not having to dig each other's ideas painfully out of the published literature, the luxury of being able to repeat a question several times (which is not possible in an ordinary conference) and of not having to understand the first time, and the luxury of having ideas repeated with different emphasis and in various settings and shadings.

There was not time during the session to prepare material for publication. However, it seems likely that results obtained in Copenhagen will be incorporated in two or three future papers. It is impossible at present to say when these articles will appear, as the results form only a part of several much larger research efforts currently underway.

The present report will serve to summarize the main findings at Copenhagen and some of the tentative conclusions reached. The report is subdivided according to topics covered. An attempt is made in each case to indicate briefly the status of the subject prior to the Copenhagen effort and then to discuss the subsequent progress made (if any) identifying the main contributors.

Thanks are due to the CERN Theoretical Study Division for its hospitality to the participants; to the students of Hagemann's Kollegium where three of the participants were housed; and to Professor Niels Bohr and the staff of the Institute for Theoretical Physics for providing the participants with a place to work. Special thanks are due to Wright Air Development Center for having pioneered this type of meeting and to the University of North Carolina for having undertaken the administration of it.

Bryce S. DeWitt

Chapel Hill, North Carolina,

October 8, 1957

${ }^{2}$ Participating during the last eleven days only. 


\section{Results}

\section{The theory of measurement}

In every new development in physical theory which extends or generalizes previous concepts, there arises at a certain stage a need to examine the overall self-consistency of the innovation. In the development of the quantum theory this self-consistency problem has centered around the "theory of the measurement process", since it is a special view of what is meant by a "measurement" which forms the core of the quantum innovation in physics. Moreover, each new development in the quantum theory itself has eventually necessitated a reexamination of measurement theory. Normally this reexamination comes at the end of a process of formalistic development (e.g. in electrodynamic theory). In the application of quantum mechanics to the general theory of relativity, however, the question of "measurability" has been raised even before the advent of a formalism - which, as of this date, still does not exist. Such activity as has so far taken place on this problem seems to have been mainly directed toward proving the inapplicability of quantum mechanics in the gravitational domain. If such inapplicability were really true, theoretical physicists would, of course, be saved a great deal of labor. In view of the formidable nature of the task of uniting the quantum and general relativity theories, one cannot, however, avoid the suspicion that such efforts may stem partly from wishful thinking.

The question of measurability was raised in Copenhagen by Professor Møller, who had some lengthy discussions on it just a few weeks previously with Professor E. P. Wigner of Princeton. In approaching the problem of measurement, Wigner has begun by trying to construct the most efficient measuring devices which the principles of quantum mechanics allow. In general relativity theory the pertinent measurement consists of a determination of the invariant interval between space-time events, which Wigner showed could always be performed by means of clocks. Wigner's problem therefore reduced initially to the problem of constructing the most efficient possible clocks. Having done this Wigner then wishes to proceed to the study of the influence of such clocks themselves on the objects being measured. Preliminary reports by his student, Dr. H. Salecker, presented at the Chapel Hill Conference in January $1957^{3}$, indicated that very serious limitations are imposed on the measurability of the gravitational effects of masses as small as the heavier elementary particles - a result of considerable significance for the validity of any attempt to quantize general relativity.

While Professor Møller was in general agreement with Wigner's analysis of clocks (aside from some purely technical modifications which he introduced in the work sessions and which it is unnecessary to reproduce in this report) he was utterly unable to follow Wigner's subsequent implications in regard to the limitations on the measurement of gravitational effects of small masses. These implications hinge on the determination of the perturb-effect of the clocks and their registering devices, but the picture which he has so far presented is by no means clear.

Professor Møller quoted a statement made by Professor Bohr after finishing his famous "measurement" paper with L. Rosenfeld, viz.

During the course of our study of the quantum limitations on the measurability of the electromagnetic field we made every possible mistake. In each case, in order to extricate ourselves, we had to go back and look at the formalism!

In Professor Møller's view it will be equally necessary, in the case of quantum gravidynamics, to have a valid formalism available, before one can make any reliable statements about the measurement problem. And even when such a formalism is finally

${ }^{3}$ See "Conference on the Role of Gravitation in Physics" WADC Technical Report 57-216. 
found, Møller does not believe Wigner's implications will be substantiated. On the contrary, Møller believes that the gravitational effect of small masses can always be measured in principle if one simply has an arbitrarily long time at one's disposal.

DeWitt and Misner adduced the following arguments in support of this stand:

Consider first a classical test particle of mass $m$ which is initially at rest at the point $x_{0}$ in a gravitational potential $\varphi$. At the time $t$ the position of the particle will be

$$
x=x_{0}-\frac{1}{2} \frac{\partial \varphi}{\partial x} t^{2}
$$

The gravitational field strength is given by $\partial \varphi / \partial x$ and can be immediately determined by a measurement of $t$ and $x$. If, however, the particle is subject to quantum laws its initial position and velocity ${ }^{4}$ are subject to uncertainties related by

$$
\Delta v_{0}=\frac{\hbar}{m \Delta x_{0}}
$$

leading to a spread in the position uncertainty of amount

$$
\Delta x=\Delta v_{0} t=\frac{\hbar t}{m \Delta x_{0}}
$$

The initial position measurement may be made by a photon of momentum uncertainty $\Delta p=m \Delta v_{0}$. The resulting uncertainty in the initial time may be ignored since it is given by $\Delta t_{0}=\Delta x_{0} / c=\hbar / m c \Delta v_{0} \ll t$ (assuming $\Delta v_{0} \ll c$ ). The final position and time measurements may be made with arbitrarily high precision, using energetic photons, since the experiment is then over.

Suppose the gravitational field ${ }^{5} \varphi$ is produced by another particle of equal mass $m$. Then $\varphi=-G m / x, \partial \varphi / \partial x=G m / x^{2}, \partial^{2} \varphi / \partial x^{2}=-G m / x^{3}$, and, apart from a factor 2 involved in transforming to center-of-mass coordinates and using the "reduced mass", equation (1) will hold with $x$ interpreted as the separation distance.

Certain obvious conditions must now be satisfied in order that the gravitational field be determined from the classical equation (1), namely

$$
\max \left(\Delta x_{0}, \Delta x\right) \ll\left|x-x_{0}\right| \ll x
$$

or

$$
\max \left(\Delta x_{0}, \frac{\hbar t}{m \Delta x_{0}}\right) \ll\left|\frac{\partial \varphi}{\partial x}\right| t^{2}=\frac{G m t^{2}}{x^{2}} \ll x
$$

Another requirement, viz.

$$
\left|\frac{\partial^{2} \varphi}{\partial x^{2}}\right|\left|x-x_{0}\right| \ll\left|\frac{\partial \varphi}{\partial x}\right|
$$

or

$$
x=\frac{\left|\frac{\partial \varphi}{\partial x}\right|}{\left|\frac{\partial^{2} \varphi}{\partial x^{2}}\right|} \gg\left|x-x_{0}\right|
$$

which says that $|\partial \phi / \partial x|$ must not change appreciably during the course of the motion, is then automatically satisfied.

\footnotetext{
${ }^{4}$ Editors' comment: $v_{0}$

${ }^{5}$ Editors' comment: DeWitt means "gravitational potential".
} 
Introducing dimensionless quantities $\Delta \xi_{0}, \xi, T$, by the relations

$$
\Delta x_{0}=\frac{\hbar^{2}}{G m^{3}} \Delta \xi_{0}, \quad x=\frac{\hbar^{2}}{G m^{3}} \xi, \quad t=\frac{\hbar^{3}}{G^{2} m^{5}} T
$$

one may rewrite these conditions in the form

$$
\max \left(\Delta \xi_{0}, \frac{T}{\Delta \xi_{0}}\right)=\alpha \frac{T^{2}}{\xi^{2}}=\alpha \beta \xi
$$

where $\alpha, \beta \ll 1$. We may distinguish two cases.

$$
\begin{gathered}
I: \alpha \beta \xi=\Delta \xi_{0}>\frac{T}{\Delta \xi_{0}}=\frac{1}{\alpha} \sqrt{\frac{\xi}{\beta}} \\
I I: \alpha \beta \xi=\frac{T}{\Delta \xi_{0}}>\Delta \xi_{0}=\frac{1}{\alpha} \sqrt{\frac{\xi}{\beta}}
\end{gathered}
$$

Both cases lead to $\xi>\frac{1}{\alpha^{4} \beta^{3}}$. Writing

$$
\xi=\frac{1}{\alpha^{4} \beta^{3} \gamma^{2}}, \quad T=\sqrt{\beta \xi^{3}}=\frac{1}{\alpha^{6} \beta^{4} \gamma^{3}}
$$

where $\gamma<1$, we have $\Delta \xi_{0} / \xi=\alpha \beta$ in case I and $\Delta \xi_{0} / \xi=\alpha \beta \gamma$ in case II. Equations (12) show that the measurement can be performed provided simply that $x$ and $t$ be chosen large enough. Since, however, $t$ varies inversely as the fifth power of $m$, the required values quickly become fantastic for small $m$. The lowest values are obtained with the choice $\gamma=1$ for which cases I and II coalesce.

There is one more condition which must be satisfied: the initial photon must have an energy $\ll m c^{2}$ in order that its own gravitational influence be negligible. This simply means that $\Delta x_{0}$ must be much larger than the Compton wavelength corresponding to $m$. Thus

$$
\Delta x_{0} \gg \frac{\hbar}{m c} \quad \text { or } \quad \Delta \xi_{0}=\frac{1}{\delta} \frac{m^{2}}{\mu^{2}}, \quad \delta \ll 1
$$

where

$$
\mu=\sqrt{\frac{\hbar c}{G}} \approx 10^{-5} \mathrm{~g}
$$

Choosing $\gamma=1$, so that

$$
\frac{1}{\delta} \frac{m^{2}}{\mu^{2}}=\Delta \xi_{0}=\frac{1}{\alpha^{3} \beta^{2}}, \quad T=\frac{1}{\alpha^{6} \beta^{4}}=\frac{1}{\delta^{2}} \frac{m^{4}}{\mu^{4}},
$$

we may distinguish two further cases:

$$
A: m<\mu
$$

Here we must take $\delta=\frac{m^{2}}{\mu^{2}} \alpha^{3} \beta^{2} \lll 1$.

$$
B: m>\mu
$$


Here we may choose ${ }^{6}$ freely (subjected to the restriction $\delta \ll 1$ ) and then take $\alpha^{3} \beta^{2}=$ $\frac{\mu^{2}}{m^{2}}$.

The absolute minimum values of $x$ and $t$ for measurability are given by $\alpha=\beta=1$ in case $\mathrm{A}$ and $\alpha=\beta, \delta=1$ in case $\mathrm{B}$. Thus

$$
\begin{aligned}
& x \gg \frac{\hbar^{2}}{G m^{3}}, \quad t \gg \frac{\hbar^{3}}{G^{2} m^{5}} \text { when } m<\mu \\
& \left.\begin{array}{l}
x \gg \frac{\hbar^{2}}{G m^{3}}\left(\frac{m^{2}}{\mu^{2}}\right)^{\frac{7}{5}}=\left(\frac{\hbar^{3} G^{2}}{m c^{7}}\right)^{\frac{1}{5}} \\
t \gg \frac{\hbar^{3}}{G^{2} m^{5}} \frac{m^{4}}{\mu^{4}}=\frac{\hbar}{m c^{2}}
\end{array}\right\} \text { when } m>\mu
\end{aligned}
$$

There is never any difficulty in satisfying the latter set of conditions. In fact, when $m \geq \mu$ the measurements can be performed entirely classically, without regard to quantum effects. To see how fantastic the figures can be, on the other hand, when $m<\mu$, consider the case of protonic mass: $m \approx 10^{-24} \mathrm{~g}$. Then

$$
\begin{gathered}
\frac{\hbar^{2}}{G m^{3}} \approx 10^{25} \mathrm{~cm} \approx 10 \text { million light years } \\
\frac{\hbar^{3}}{G^{2} m^{5}} \approx 10^{53} \mathrm{sec} \approx 10^{46} \text { years }
\end{gathered}
$$

Nevertheless there is no point of principle which makes such measurements impossible. There is no difficulty in constructing a clock accurate enough to measure such large time intervals, and massive enough so that its own position uncertainty does not mask the position measurement of the masses $m$ during this time. Finally the perturbing effect of the clock's gravitational field may be eliminated by incorporating the clock in a huge spherical shell of ${ }^{7}$ mass density completely surrounding the experiment.

\section{Topological problems}

Although no questions of principle seem to disallow the union of the quantum and general relativity theories (on the contrary, there are strong reasons for attempting such a union) there are nevertheless many difficulties to be faced before it can be successfully brought about. In all previous cases, the classical behavior of any physical system possessing a classical analog has been thoroughly understood before the quantum theory was applied to it. In the case of the gravitational field, however, this classical behavior itself is not well understood. The classical gravitational field is exceedingly complex and rich in possibilities as yet unexplored.

Among the more curious of these possibilities is that which arrises because the gravitational field determines the space in which it itself acts - and in particular, the topology of that space. The manifold of possible topologies is infinite, just as the manifold of possible fields within each topology is infinite. It is clearly of interest to examine at least some of the typical topological situations which can arise.

Dr. Misner, working with Professor Wheeler's group at Princeton, had already during the previous two or three years examined some of the simplest topological possibilities. At Copenhagen he reported in some detail on the results of this work, leaving a sharpened awareness of its importance and significance in the minds of the other participants. As the simplest special topology Misner called attention

\footnotetext{
6 Editors' comment: $\delta$

7 Editors' comment: uniform
} 
to the Schwarzschild metric. With the use of conformal coordinates it is easy to see that the simplest interpretation of the Schwarzschild metric is that of a two sheeted topology (cf. Einstein and Rosen) with no singularities. In emphasizing another aspect of this metric, Misner pointed out that the Schwarzschild metric is that which describes the gravitational field of a "mass", which is of course familiar to everyone. The special point which Misner made, however, was that from a certain point of view there is no mass; the appearance of a "source" of the gravitational field is obtained by a topological trick. By combining the Maxwell field with the Einstein field Misner had (as reported at the Chapel Hill conference) also been able to represent sources of the electromagnetic field (i.e. "charges") by means of topological tricks.

Dr. Deser raised the question as to whether the sources of other fields (e.g. nuclear fields) can also be represented by topological tricks. He and Misner investigated the scalar meson fields and, in the massless case, found that the topological representation is again possible. In the case of non-vanishing mass the Einstein equations are difficult to solve, but it again appears in principle possible to use topological tricks to represent sources. In the study of the latter problem Deser and Misner had the help of Dr. A. Komar who had been a member of the Institute for Theoretical Physics during the past year, was still in Copenhagen, and was studying precisely the problem of the interaction of scalar and gravitational fields.

(In view of recent work of Klauder and Wheeler it appears that similar possibilities do not occur for spinor fields (Klauder and Wheeler 1957). That is, topological tricks cannot be employed to represent sources of spinor fields.)

Misner also outlined the situation in regard to more complicated topologies, in which several "sources" are present. Conformal coordinates can again be used at the initial instant, for both the neutral mass and charged mass cases, and Misner has shown that fairly simple and intuitively reasonable metrics which satisfy the Fourès equations for the Cauchy initial value conditions can be introduced for these topologies. The interest of these topologies is that they are dynamically active, i.e. they move. With several sources one can have a many-sheeted topology, a two-sheeted topology, or a "wormhole" topology. (See report of the Chapel Hill Conference.) The initial value conditions are easiest to "mock up" for the wormhole topology, simple monopole terms being sufficient, whereas for the two-sheeted topology multipole terms of all orders are required. However, the theory of both types of topologies is now sufficiently well advanced so that one could probably put a two-source problem on a high-speed computing machine without excessive difficulty. This may be done in the next year or so and would be of very great interest, especially the determination of what happens in very close encounters between sources.

In view of the apparent tractability of the multi-source problem, DeWitt and Laurent were unable to see why Misner had seemed to have proved two years ago the impossibility of a particularly simple type of "wormhole" dynamics: the case of a simple toroidal structure dominated by a closed electric field. Misner therefore took the problem up again and found an error in his previous work. He found, contrary to his original finding, that the toroidal dynamics is indeed possible, and, moreover, he was able to solve the differential equation for the toroidal expansion in closed form.

Another aspect of the topological problem arises from the following considerations: Sources of fields may be represented by topological tricks. On the other hand, topologies may be completely described by the metric. To what extent, therefore, can fields quite generally be described purely by metric? G. Y. Rainich showed many years ago that the electromagnetic field could be completely described by metric quantities (Rainich 1925). Its stress tensor leaves a sufficiently characteristic imprint on the metric for the complete details of the field itself to be deduced. Misner has worked out the details of the necessary deciphering process more completely than can be found in Rainich's work. At Copenhagen he and Deser investigated the feasibility 
of a deciphering procedure for a scalar field as well, and readily found one. They were able to show, moreover, that one can also handle several fields at a time, i.e. that various field can be disentangled from one another and identified in detail merely through the combined stress tensor. One cannot, however, disentangle two fields of the same variety (i.e. identical), and the problem becomes difficult when there are interactions between the various fields. It is possible, owing to the work of Takabayasha (various articles in Prog. Theor. Phys. and Il Nuovo Cimento in recent years) who has arrived at a complete description of spinor fields by means of hydrodynamic quantities, that the work of Deser and Misner can also be extended to spinor fields.

\section{Feynman quantization}

Having a keen appreciation of the great amount of work which remains to be done in the purely classical aspects of gravitational theory, it was with considerable soberness that the participants approached the main problem of the work sessions - the quantization problem. It was recognized that the successful solution of this problem will have to overcome many obstacles. The most direct frontal attack on the problem is that suggested by Professor Wheeler, who proposes simply to write

$$
\int \exp \left[\frac{i}{\hbar}(\text { Einstein action })\right] d \text { (field histories) }
$$

in order to obtain the invariant transformation function for the gravitational field. Dr. Misner, working with Wheeler, has perhaps done more than anyone else in pushing the investigation along these lines, which for the cases of simpler field theories, were laid down originally by R. P. Feynman.

The Feynman approach, because of its directness, runs quickly into a number of difficulties, among the chief of which are: (1) the necessity to discover or define an appropriate invariant "measure" with respect to which to carry out the Feynman integration; (2) the necessity of defining invariant state functionals to serve as bounds in the Feynman integration; and (3) the necessity of finding appropriate operators in terms of which to define physically significant observables and boundary conditions. The latter two difficulties arise in any approach to quantization, but the first difficulty is of special immediacy and importance in the Feynman method.

By introducing the notion of "homogeneity group" Misner has succeeded in defining a possible invariant measure for the Feynman integration. In Misner's argument no use is made whatsoever of the dynamical aspect of the gravitational field as exemplified by its Lagrangian; the whole rests solely on arguments of general invariance. This is by no means an unreasonable point of view and certainly does not mean, for example, that the measure cannot also be determined in fact from the Lagrangian. For the precise form which the Lagrangian has depends on properties of general invariance. Nevertheless, since appeal to the Lagrangian is essential according to the canonical viewpoint (see next section) it is of importance to see whether or not the two viewpoints are in harmony.

Dr. Laurent, at Copenhagen, developed an argument based on local linearization of the Einstein equations and the use of "hyper delta functions" in functional space to show that the Lagrangian indeed leads to Misner's "measure". There are still some uncertainties in Laurent's argument, chiefly concerning the admissibility of certain types of state functionals, and in the view of DeWitt, who has been developing a rigorous canonical approach (see next section), the problem is still not settled. Misner's measure has the virtue that it becomes singular when the metric tries to change its signature, thereby imposing a natural barrier to the extension of the Feynman 
integration over nonphysical field histories. Misner's measure, however, allows physical conditions to be specified on surfaces which are not necessarily space-like. On the other hand, DeWitt has obtained a preliminary measure which not only becomes singular when the metric tries to change signature but also becomes singular when the metric attempts to change the character of a surface (on which physical specifications have been made) from space-like to time-like.

Considerable discussion developed on the question of the appropriate field-history domain for a Feynman integration. This problem has several aspects. First, it was asked whether the existence of a natural barrier in Misner's or DeWitt's measure necessarily excludes integration over nonphysical field histories. It seems that the propagation amplitude will damp out anyway very rapidly in such domains, owing to the classical action becoming imaginary instead of real, and integration over such regions may in fact be necessary in order to obtain correct analytic behavior of asymptotic wave functionals ${ }^{8}$ just as quantization of simple systems necessitates penetration of the wave function into classically nonphysical regions (barrier penetration).

Secondly, the question of topologies was raised. If one be permitted to integrate over nonphysical domains must one not also consider the possibility of integrating over various topologies? Professor Wheeler has championed the answer "yes" to this question, although in view of the infinity of possible topologies the prospect is slightly terrifying. The question itself has special aspects. For example, suppose it has been decided that integration over nonphysical signatures is to be excluded. There still remains a question as to the existence of 4-dimensional domains of constant signature which are bounded by two or more positive definite 3 -spaces having different topologies, or whether peculiar topologies can exist as intermediate states to simple topologies. Misner plans to carry out extensive researches on these questions with the aid of the Princeton topologists. The problem, however, is exceedingly difficult, most of its aspects being to date unsolved or unknown. This becomes particularly obvious when one recalls that the bounding 3-spaces may be topologically different merely in virtue of "knottedness", and even the "knot problem" for closed two dimensional domains has not been solved.

It was conjectured in Copenhagen that the topological integration problem may bear some slight analogy to a boundary condition problem. Consider the quantum theory of a simple particle in a box. In order to treat this system by the Feynman method it is necessary not only to integrate over all direct paths between two space time points but also to sum over the infinity of classically distinct types of paths corresponding to repeated reflections of the particle from the walls of the box. Only then will the wave function satisfy the necessary boundary condition of vanishing at the box wall. In some vaguely similar sense the summation over the infinity of topologically distinct gravitational field histories may correspond to a special boundary condition which may or may not be satisfied in Nature.

\footnotetext{
8 i.e., "analytic" in the sense of the theory of functions of a nondenumerable infinity of complex variables. Another, more startling example of penetration into a nonphysical region is provided by the strict probabilistic interpretation of the scalar wave equation (KleinGordon). Using the relativistic position operator defined by Newton and Wigner (1949) one can show that the correct propagation function for the position operator is not the invariant delta function $\Delta\left(x-x^{\prime}\right)$ which vanishes outside the light cone, but rather the time derivative of the function $\Delta^{+}=\frac{1}{2}\left(\Delta-i \Delta^{(1)}\right)$ which does not vanish outside the light cone, although it does damp out rapidly whithin a Compton wavelength for the light cone. This, of course, is due to certain nonlocal features of relativistic particles when viewed from the traditional quantum standpoint. And here we have the first hint that vaguely similar, although profoundly deeper, nonlocal features will make this appearance in quantum gravitational theory.
} 
Because of the formidable nature of the topological problems there is no doubt that any calculations which may eventually be made in quantum gravitational theory will initially assume both constant signature and constant topology. In virtue of the rich potentialities which variable topologies provide, however, one would be exceedingly rash at this stage to assert the necessity of such constancy.

Occupying a position complementary to that of the Feynman theory is the Schwinger quantization method. Just prior to the Copenhagen gathering Professor DeWitt had discussed with Professor Schwinger in Lille (Mathematical Congress) the question of applying his (Schwinger's) methods to the gravitational field. One major difficulty facing Schwinger's technique is that he deals with operators from the outset, and therefore must know a great deal about the commutation relations (i.e., dynamics) of the system he is dealing with already in advance. This becomes particularly troublesome when the theory if nonlinear as Einstein's is. Schwinger's suggestion was to work with the Palatini formalism which introduces the affine components along with the metric as fundamental field quantities. The appropriate Lagrangian is then linear in the field derivatives and the commutation difficulties arising from quadratic occurrences of the derivatives are thereby eliminated - according to Schwinger.

This possibility was considered at Copenhagen, but apart from a curious ambiguity which was noted in setting up gravitational interactions with other field within the Palatini formalism, work was not pushed very far in this direction. The trouble with using Palatini à la Schwinger is that the difficulties are simply postponed to a later stage, viz. the stage where one must begin to look at the constraints, which, in the Palatini formalism, are of the "second class" as well as the "first" (Dirac 1950). Within the Schwinger formalism constraints must be handled ad hoc. Each type of system must be given a special treatment, and the gravitational field will require the most special (i.e., difficult) treatment of all. It seemed highly unlikely to the participants at Copenhagen that Schwinger's methods would really prove useful in the search for the hidden path to a quantum theory of gravitation. On the other hand, there is no doubt that when a quantum gravitational formalism is finally found Schwinger will be on hand to reformulate it.

\section{Canonical quantization}

Although the Feynman viewpoint enables one to obtain rapid and far reaching insights into the quantum theory of a given system, it is less successful in separating and isolating the technical difficulties which arise when calculations are attempted on the system. There is a tried and (so far) true method of quantizing any system which possesses a classical analog. This is the so-called canonical quantization method laid down originally by Schrödinger, Heisenberg and Pauli. About ten years ago Professor P. G. Bergmann at Syracuse University took up the problem of applying this method to the gravitational field. It is he who first exposed in detail the main difficulties besetting the problem and who introduced the notion of "true observables" (coordinateinvariant quantities) and their relation to the primary and secondary constraints of the system (Dirac 1950).

At Copenhagen some new progress was made on Bergmann's problem by Professor DeWitt. During the preceding year DeWitt had been engaged in an effort to develop mathematical tools for the analysis of the constraints which arise in a canonical system as a result of the existence of invariance groups. His program consists of a study of the types of transformations to which the constraints themselves may be subjected, the types of Lie algebras generated by the constraints, the interrelation of the constraints, the Lagrangian and the "true measure" (i.e. for Feynman quantization 
of the system), and the elimination of the constraints from the quantum theory implying a simultaneous discovery of the "true observables" and resolution of the factor-ordering ambiguity.

At Copenhagen DeWitt succeeded in rigorously isolating half of the non-observable variables, those corresponding to the primary constraints. The chief problem was to find a canonical transformation which transforms the primary constraints into pure momenta. Professor J. L. Anderson of Stevens Institute of Technology had previously conjectured the possibility of such a transformation, but it had not up to this time been carried out by anyone. In order to find the transformation it was necessary to solve a set of simultaneous variational differential equations of a type which will continue to characterize DeWitt's work for some time to come. DeWitt found the solution only after several days of seemingly fruitless effort - an indication of the difficulty of the whole technical problem. However, his solution is rigorous and he has not yet been forced to resort to a successive approximation scheme.

DeWitt is now able to turn his attention to the secondary constraints, by far the most interesting quantities in the theory and to the understanding of which the major effort is now being devoted. It is impossible at the present time to predict when this work will be finished or when it will have reached a form suitable for publication. DeWitt has incidentally shown that Anderson's conjecture about the transformability of constraints is generally wrong. It is correct only if the Lie group generated by the constraints is Abelian, and it happens that the group generated by the primary constraints can be shown to be necessarily Abelian. The important question raises itself as to whether the group generated by the secondary constraints is also Abelian or not. If it were, and if DeWitt could find the transformation which changes the secondary constraints into momenta, then the problem of formally quantizing general relativity would at last be solved, and one could begin to compute some physical quantities. Unfortunately, the question is not easily answered. Strong arguments can be adduced on one side to prove that the group is Abelian, but equally strong arguments can be found on the other side to prove that it is not. These contradictory conclusions stem from reasoning by analogy with finite Lie groups. The Lie groups of general relativity are, however, infinite - and very little is known of the mathematical properties of infinite Lie groups. Nevertheless, Professor W. Pauli of Zürich has conjectured that infinite Lie groups will eventually turn out to be of greater importance in physics than finite parameter groups have ever been (Pauli 1957). This is mentioned to indicate the significance of the problem which still lies ahead.

Of importance in DeWitt's work is the use of the parameter formalism of P. Weiss (Weiss 1938). The parameter formalism has the following principal advantages:

1. The parameters serve to label the points in the underlying space-time manifold once and for all. They are not subject to transformation. The so-called "coordinates" have then a much greater functional similarity to the metric components, than in the ordinary formalism and, like the metric components, can be imposed in an arbitrary way on the underlying manifold (subject only to the secondary constraints).

2. The change of viewpoint achieved by the use of parameters permits one to perform functional operations and transformations which would otherwise be impossible. This promises to greatly simplify the work of transforming the secondary constraints into momenta (if this is at all possible).

To convey something of the utility of the parameter formalism some of DeWitt's results will be recorded here:

One of the parameters is chosen as the independent parameter $T$ of a canonical formulation. Since the choice is arbitrary no invariance is destroyed. Surfaces $T=$ constant are then regarded as space-like and a normal vector density $\ell_{\mu}$ is introduced. 
After isolation of the non-observable variables corresponding to the primary constraints, DeWitt finds a Schrödinger equation of the form

$$
\varphi_{\mu} \equiv \lambda_{\mu}+\mathcal{H}_{\mu}=0
$$

where

$$
\begin{aligned}
\mathcal{H}_{\mu} \equiv & \frac{1}{2} \ell_{\mu} G_{\alpha \beta \gamma \delta} \Pi^{\alpha \beta} \Pi^{\gamma \delta}-2 \ell^{-4} \ell_{\mu} \ell^{\gamma} \Pi^{\alpha \beta} \nabla_{\gamma \alpha} \ell_{\beta}-\ell^{-2}\left(2 \ell^{\alpha} \Pi^{\beta \gamma}\right. \\
& \left.-\ell^{\gamma} \Pi^{\alpha \beta}\right) \nabla_{\mu \nu} g_{\alpha \beta}-\nabla_{\mu \alpha}\left(\Pi^{\alpha \beta} a_{\beta}\right)+Y_{\mu} \\
G_{\alpha \beta \gamma \delta} \equiv & g^{-\frac{1}{2}} \ell^{-2}\left(\bar{g}_{\alpha \gamma} \bar{g}_{\beta \delta}+\bar{g}_{\alpha \delta} \bar{g}_{\beta \gamma}-\bar{g}_{\alpha \beta} \bar{g}_{\gamma \delta}\right) \\
g \equiv & \left|g_{\mu \nu}\right|, \quad \ell^{2}=-g^{\mu \nu} \ell_{\mu} \ell_{\nu}, \quad \bar{g}_{\alpha \beta}=g_{\alpha \beta}+\ell^{-2} \ell_{\alpha} \ell_{\beta} \\
\ell^{\alpha} \equiv & g^{\alpha \beta} \ell_{\beta}, \quad \nabla_{\alpha \beta}=\ell_{\alpha} \frac{\partial}{\partial x^{\beta}}-\ell_{\beta} \frac{\partial}{\partial x^{\alpha}} .
\end{aligned}
$$

The $\lambda_{\mu}$ are momenta conjugate to the coordinate $\chi^{\mu}$ and the $\Pi^{\alpha \beta}$ are momenta conjugate to the remaining variables $\psi_{\alpha \beta}$. Here $\psi_{\alpha \beta}$ is a symmetric tensor with ten components, of which, however, only six are independent, and $\Pi^{\alpha \beta}$ satisfies $\Pi^{\alpha \beta} \ell_{\beta} \equiv$ 0 . In the quantum theory $\lambda_{\mu}$ becomes an operator $-i \hbar \delta / \delta x^{\mu}$ acting on the state functional $\psi[x]$ in the Schrödinger equation $\varphi_{\mu} \psi[x]=0$. The relation between $\psi_{\alpha \beta}$, $g_{\alpha \beta}$ and the variables $a_{\alpha}$ conjugate to the primary constraints $\phi^{\alpha}$ is: $g_{\alpha \beta}=\frac{1}{2}\left(\ell_{\alpha} a_{\beta}+\right.$ $\left.\ell_{\beta} a_{\alpha}\right)+\psi_{\alpha \beta}$. The $Y_{\mu}$ are rather complicated functions of the $a_{\alpha}$ and $\psi_{\alpha \beta}$, but involving no momenta.

The Schrödinger equation is, as one would expect, seen to involve the momenta $\Pi^{\alpha \beta}$ quadratically, trough the "Hamiltonian vector" $\mathcal{H}_{\mu}$. However, only the component of $\mathcal{H}_{\mu}$ parallel to $\ell_{\mu}$ is thus quadratic. The remaining three components are linear in the momenta and correspond to the redundancy in the description of space-like surfaces by means of parameters.

The secondary constraints are obtained by taking the Poisson bracket of $\varphi_{\mu}$ with the primary constraints $\phi^{\nu}$. DeWitt finds

$$
\begin{aligned}
\left(\varphi_{\mu}, \phi^{\prime \nu}\right) \equiv & {\left[\frac{1}{2} \ell^{\nu} \mathcal{H}_{\mu}+\ell^{-2} \ell^{\alpha} \Pi^{\nu \gamma} \nabla_{\gamma \alpha} \ell_{\mu}-\frac{1}{2}\left(2 g^{\alpha \nu} \Pi^{\beta \gamma}-g^{\nu \gamma} \Pi^{\alpha \beta}\right) \nabla_{\mu \gamma} g_{\alpha \beta}\right.} \\
& \left.-\nabla_{\mu \alpha} \Pi^{\alpha \beta}+\frac{1}{2} \ell^{\nu} \nabla_{\mu \alpha}\left(\Pi^{\alpha \beta} a_{\beta}\right)-\frac{1}{2} \ell^{\nu} Y_{\mu}\right] \delta\left(u-u^{\prime}\right)+\frac{\delta Y_{\mu}}{\delta a_{\nu}^{\prime}}
\end{aligned}
$$

where $u$ and $u^{\prime}$ are the labels of points on a space-like surface. The above expression involves the momenta quadratically trough the occurrence of $\mathcal{H}_{\mu}$. However, because of the Schrödinger equation, $\mathcal{H}_{\mu}$ may be replaced by $-\lambda_{\mu}$, leaving an expression involving sixteen components which are linear in the momenta. Twelve of these components are mere repetitions of the three components of the Schrödinger equation corresponding to the aforementioned redundancy in the description of space-like surfaces. The remaining four components are the secondary constraints:

$$
\begin{aligned}
\chi_{\mu} \equiv & -\frac{1}{2} \ell_{\mu} \ell^{\nu} \lambda_{\nu}+\ell^{-2} \ell^{\alpha} \ell^{\nu} g_{\mu \beta} \Pi^{\beta \gamma} \nabla_{\gamma \alpha} \ell_{\nu}-\ell^{\nu} \Pi^{\beta \gamma} \nabla_{\nu \gamma} g_{\mu \beta} \\
& +\frac{1}{2} \ell^{\nu} \Pi^{\alpha \beta} \nabla_{\nu \mu} g_{\alpha \beta}-\ell^{\nu} g_{\mu \beta} \nabla_{\nu \alpha} \Pi^{\alpha \beta}+\frac{1}{2} \ell_{\mu} \ell^{\nu} \nabla_{\nu \alpha}\left(\Pi^{\alpha \beta} a_{\beta}\right)+Z_{\mu}=0
\end{aligned}
$$

where

$$
g_{\mu \alpha} \ell^{\nu} \frac{\delta Y_{\nu}}{\delta a_{\alpha}^{\prime}}-\frac{1}{2} \ell_{\mu} \ell^{\nu} Y_{\nu} \delta\left(u-u^{\prime}\right) \equiv Z_{\mu} \delta\left(u-u^{\prime}\right) .
$$


By means of the parameter formalism the secondary constraints have been rendered linear in the momenta. This will greatly simplify the procedure of transforming these constraints themselves into pure momenta, if this is at all possible. However, the most startling feature is the occurrence of $\ell^{\nu} \lambda_{\nu}$ in the fourth secondary constraint:

$$
\ell^{-2} \ell^{\mu} \chi_{\mu} \equiv \frac{1}{2} \ell^{\nu} \lambda_{\nu}-\frac{1}{2} \ell^{-2} \ell^{\mu} \ell^{\nu} \Pi^{\beta \gamma} a_{\beta} \nabla_{\nu \gamma} \ell_{\mu}-\ell^{-2} \ell^{\mu} \ell^{\nu} \nabla_{\nu \gamma}\left(\Pi^{\beta \gamma} \psi_{\mu \beta}\right)+\ell^{-2} \ell^{\mu} Z_{\mu}
$$

A transformation which changes this component into a pure momentum will of necessity produce a functional mixing up of the coordinates $\chi^{\mu}$ with the metric components. After the "true observables" are then extracted the resulting theory will have a nonlocal character which is unique among field theories ${ }^{9}$. DeWitt is currently devoting his efforts to the study of expression (31), optimistic in his expectation that the secondary Lie group will prove to be Abelian like the primary group. However, even if this group proves to be non-Abelian all is not lost. It simply means that a (doubtless difficult) study of the classification and representations of infinite non-Abelian Lie groups will have to be undertaken.

Another advantage of the parameter formalism, noted in Copenhagen, was that it served in good measure as an aid to the participants in understanding one another. It served to fix ideas and to separate problems of general covariance from problems of constraints. By providing an explicit representation it served as an aid in translating the mathematical abstractions of Misner's work into concrete terms. For example, it enabled the other participants to understand Misner's result that the Hamiltonian operator in any "topologically invariant" theory vanishes (Misner 1957). It is not hard to see that Misner's use of the term "topologically invariant" is equivalent to using a dynamics based on the parameter $T$. But the state vector $\psi$ does not depend on $T$, and the "Hamiltonian" corresponding to $T$ consequently vanishes. True dynamics is obtained only through the constraint equation $\varphi_{\mu} \psi[x]=0$.

\section{Approximation methods}

Since a definitive formulation of quantum gravitational theory is yet to be achieved, it may seem somewhat premature to consider the technical problems of computation at this stage. Nevertheless, attention was given to this matter at Copenhagen, partly with an eye toward seeing what difficulties one may expect to encounter in the future.

In the view of Dr. Deser, ordinary expansion methods (in hoped-for future calculations) will be worthless. He points out that in general relativity even a matter Lagrangian will have, strictly speaking, no "free-particle" part, because the "coupling" to the gravitational field enters already in the kinetic energy in a multiplicative fashion. He maintains that the special character of this fundamentally new mode of coupling will make itself felt most critically at high energies and forbid any approximations from "free-particles" just at the point where the divergence difficulties

\footnotetext{
${ }^{9}$ Here the nonlocality of the quantized gravitational field, hinted at in the preceding section, becomes explicit. A curious possibility arises at this point: The only momenta quadratically involved in the Schrödinger equation (23) are the $\Pi^{\alpha \beta}$. However, because the extraction of the true observables will involve the coordinates $x^{\mu}$ themselves in a peculiar way it may happen that the Schrödinger equation, after extraction, will involve also the $\lambda_{\mu}$ quadratically. The physical meaning of such a situation is not at all clear, just as the original interpretation of the relativistic wave equation, involving second time derivatives, was not clear. It might happen that a "square root" procedure analogous to that used in the development of the Dirac equation would have to be adopted, thus allowing both spinors and Fermi statistics to come into physics from Einstein's theory from a curious back door.
} 
of modern field theories now exist. Dr. Misner also expresses a similar view, calling attention to the fact that if gravitation is to occupy a significant place in modern physics, it can do so only by being qualitatively different from other fields. As soon as we assume the gravitational field to behave qualitatively like other fields we find that it is quantitatively insignificant. It is in its qualitative difference that its very special importance lies.

It is fair to state that these points of view stem largely from the philosophies of Professors Klein and Wheeler respectively. In the qualitative uniqueness of the gravitational field Klein hopes to find a mechanism for "smearing out the light cone" and thus softening the singularities of relativistic quantum field theory. Wheeler on the other hand looks to the richness of topological possibilities in general relativity to characterize the uniqueness of the gravitational field. For Wheeler the vacuum is in a state of turmoil - a "foam like" structure with an exceedingly complex topology. This view of the vacuum prevents one from basing an approximation procedure on an assumption of Euclidean topology in zeroth order.

The computational suggestions of Wheeler and Misner have not progressed beyond the most rudimentary level of dimensional considerations. Deser's suggestions are hardly any further advanced. However, Deser is at least able to write down an explicit expression which make some of the difficulties explicit. Employing Feynman's functional integration technique he obtains the following approximate formal expression for the propagation function of a relativistic particle modified by its gravitational interactions:

$$
\Delta\left(x, x^{\prime}\right) \sim N^{-1} \int \Delta\left(x, x^{\prime}, g_{\mu \nu}\right) e^{\frac{i}{\hbar} A}\left[\delta g_{\mu \nu}\right]
$$

where $N$ is a normalization factor, $A$ is the gravitational action and

$$
\Delta\left(x, x^{\prime}, g_{\mu \nu}\right) \sim\left[\int_{x}^{x^{\prime}}\left(g_{\mu \nu} d x^{\mu} d x^{\nu}\right)^{\frac{1}{2}}\right]^{-2}
$$

the latter integration being taken along a geodesic. Making still further approximations one may write $A=\frac{1}{G} \int g_{\mu \nu} \square^{2} g_{\mu \nu} d^{4} x$ and, for $x$ near $x^{\prime}$,

$$
\Delta\left(x, x^{\prime}, g_{\mu \nu}\right)=\left[g_{\mu \nu}\left(x_{\mu}-x_{\mu}^{\prime}\right)\left(x_{\nu}-x_{\nu}^{\prime}\right)\right]^{-1}
$$

so that

$$
\Delta\left(x, x^{\prime}\right) \sim N^{-1} \int \frac{e^{\frac{i}{\hbar G} \int g_{\mu \nu} \square^{2} g_{\mu \nu} d^{4} x}}{g_{\mu \nu}(x)\left(x_{\mu}-x_{\mu}^{\prime}\right)\left(x_{\nu}-x_{\nu}^{\prime}\right)}\left[\delta g_{\mu \nu}\right]
$$

One might now hope that by studying this formal functional integral one would gain certain insights as to valid approximation schemes entirely different from the usual expansion methods. For certain systems, in fact, this is occasionally possible, by treating the Gaussian forms into which the functional integral can be cast, as ordinary integrals. Unfortunately the integral above is not Gaussian as it stands and any attempt to obtain an asymptotic series by expanding the denominator fails to remove the singularity of the propagation function, which Deser shows by other arguments must be absent. It would seem that here is an example where the special features of the Feynman quantization technique prove to be of little advantage - at least as far as practical calculations are concerned.

At Copenhagen Deser and Laurent devoted considerable effort, but without much success, toward developing an approximation scheme, or at least the framework of one, based on Deser's functional integrals combined with the observation that in any quantum gravidynamical calculation $\hbar$ and $G$ always occur together in the product $\hbar G$, and hence that the classical limit $(\hbar \rightarrow 0)$ and the Euclidean limit $(G \rightarrow 0)$ occur together. 
To DeWitt this observation was strong evidence that, contrary to the opinions of the other participants, ordinary expansion procedures should be excellent. In his view the unique features - in particular, the non-locality - of the gravitational field must exist already in the classical theory. Once a canonical formalism is completely developed quantization should be straightforward. No agreement was reached on these points, and there the matter rests.

During the course of the Copenhagen gathering DeWitt obtained a result which, if it had been just the opposite, would have had a direct bearing on the matter. The investigation leading to this result actually arose initially from quite another source and not out of an effort to refute Deser's stand. It occurred to DeWitt that one might be able to find an appropriate functional transformation from the $x^{\mu}$ and $g_{\mu \nu}$ over a space-like surface ${ }^{10}$ to another 14 -fold, three dimensional continuum of quantities which would render the Einstein equation linear! Such a possibility caused DeWitt to fear that all his previous work might prove to be a great joke - that his carefully developed procedures might, in fact, be leading him steadily (however slowly) to such a linearizing transformation, which would then imply an isomorphism between Einstein's theory and a linearized theory. If this were true DeWitt could have saved himself a lot of work by looking for such a transformation at the outset. The basis for DeWitt's notion was not entirely idle or trivial. There are certain classes of nonlinear differential equations which can be rendered linear by transforming to new independent variables which are functions of both the original dependent and independent variables. Certain of the equations arising in the derivation of the wellknown exact solutions of Einstein's equations are of this form. However, when DeWitt set up the variational differential equations which would have to be satisfied by such a functional transformation he found, by an expansion procedure, that they were mutually incompatible. Hence no such transformation exists.

Had such a transformation existed then Deser would have had no ground to stand on. The appropriate procedure in calculations with the gravitational field would obviously be to proceed just as with any other field. This would not mean that gravitation would fail to retain its unique nonlocal features, for the functional transformation would still mix up the $x^{\mu}$ and the $g_{\mu \nu}$ and hence introduce non-locality into the matter interactions. Even though the transformation does not exist, and Deser's stand remains therefore unrefuted, DeWitt's stand is not thereby imperiled. DeWitt maintains that the non-locality is still quite real - even classically - and that the impossibility of linearization simply means that the true observables of the gravitational field really do suffer self-interactions, trough a coupling which is also, presumably, nonlocal.

\section{Some special problems}

Very frequently during the Copenhagen work sessions the participants found themselves confronted by a constantly recurring difficulty, namely the inadequacy of the ready-at-hand concepts of other field theories when dealing with the gravitational field. Often an argument which seemed to be proceeding smoothly would suddenly find itself enmeshed in a contradiction, which on further analysis generally proved to be the result of the inapplicability of some idea or other which had always proved valid in previous contexts. This situation can be illustrated by a simple example, viz. the idea of radiation damping.

Consider first an electrically charged particle scattering electromagnetic radiation. An incoming electromagnetic wave causes the particle to being oscillating.

\footnotetext{
10 For the consideration of such a possibility, use of the parameter formalism is essential. Without the parameter formalism the functional transformations would have to be restricted to the $g_{\mu \nu}$ alone, and it is easy to show that no linearization is possible within this framework.
} 
The oscillation of the particle causes it to emit radiation of its own. But this emitted radiation reacts back on the particle, causing it to lag slightly behind the motion it would have if it did not radiate. This effect becomes more pronounced the higher the frequency of oscillation, causing the scattering cross section which is constant at low frequencies to diminish somewhat at very hight frequencies. Accompanying the lag in the particle's motion is also a net acceleration of the particle in the direction of the incoming radiation, representing the effect of radiation pressure. The scattering itself may be regarded as an interference effect between the incoming primary radiation and the emitted secondary radiation. As the lag of the particle becomes more pronounced (at higher frequencies) the interference effect becomes less efficient and the scattering cross section (and, in fact, the oscillatory amplitude of the particle) is "damped".

At first sight one would suppose that a similar phenomenon should occur in general relativity. One simply replaces electromagnetic waves by gravitational waves and the electric charge by the particle mass. For this type of a scattering computation the weak field approximation should be eminently suitable, since cross sections are normally amplitude independent. If one proceeds with the calculation, however, one finds that, in the weak field approximation, there is no scattering at all. The geodetic motion of a particle in a weak gravitational radiation field is simply uniform rectilinear. From a more sophisticated viewpoint, of course, this is not surprising. The left hand side of the Einstein weak-field equation has identically vanishing divergence (linearized Bianchi identities). On the right hand side stands the particle stress tensor, and this must have identically vanishing divergency too. But this happens only when the particle motion is uniform rectilinear. In order to obtain real scattering effects one must proceed to at least one stage beyond the weak field approximation. But here one runs into great complications because of the nonlinearity of the field equations.

The nonlinearity of the gravitational field equations is, of course, the mechanism by which Einstein's theory manages to conserve all forms of energy, including gravitational. The last point is important. In the informal discussions, replete with ready-athand concepts, which characterized the Copenhagen work sessions, it was very easy to run into a contradiction if one did not take care to keep close track of the energy contained in the gravitational field itself. But the gravitational field energy is a very elusive quantity. In the first place, it cannot be localized. In the second place, there are perfectly well behaved systems (e.g., closed universes; cylindrical gravitational waves) for which it seems curiously indefinable, even in the large. These problems, which remain unsolved today, were encountered at a very early phase in nearly every discussion.

Returning to the discussion of radiation damping, in order to complete the picture, we may next point out that there is another method of approach to the problem which is completely unavailable in other field theories. Since the choice of coordinates in general relativity is entirely arbitrary one may, if one likes, view the particle as being always at rest instead of oscillating. This suggests the natural approach of regarding the scattering particle as a Schwarzschild singularity - or, better, as the connecting link in a two-sheeted topology - and of treating the incoming radiation as a small perturbation on the Schwarzschild metric. The appropriate mathematics has already been worked out by Professor Wheeler and Dr. T. Regge (to be published). However, this completely transforms the problem. It becomes like a problem of scattering from a fixed potential, and it is very difficult to see how a phenomenon like radiation damping can make its appearance even in an exact solution. Or, rather, it is quite clear that although a Heitler integral equation can be set up for the scattering problem, the behavior of the phase shifts will have little relation to that in the electromagnetic case, and represent much more subtle effects.

Another aspect of the problem may be pointed out. First, let us note that the above discussion of the electromagnetic case was a bit hasty. If one tries to solve the problem 
rigorously, using a point particle so as to maintain relativistic invariance, one runs into a self-energy divergence difficulty. This can be circumvented by the method of Dirac which is basically a classical mass renormalization prescription (Dirac 1938). In the renormalized theory, however, the oscillating particle does not suffer a phase lag at hight energies, but a phase advance instead (so-called "pre-acceleration") ${ }^{11}$. In the gravitational case, with the use of the Schwarzschild metric, the renormalization problem does not arise. The mass of the particle is already determined by the metric at large distances. A new flexibility in boundary conditions appears, however. Boundary conditions must be specified on both topological sheets, and one has the possibility of allowing energy to leak from one sheet to the other.

\section{Conclusion}

The example of the preceding section shows in a simple way how radically different the gravitational field is from other fields. Although the participants repeatedly stressed this fact, they were nearly as often shocked or tricked by it, almost as if they didn't really believe it. In some sense the heart of the matter lies in the curious position of the concept of energy in general relativity. On the one hand the invariance of the theory leads to strong conservation laws, among which one expects the law of conservation of energy. On the other hand the very concept of energy somehow seems to dissolve. The participants agreed that this concept needs a thorough study and review. One is tempted to say that once the concept of energy is understood, everything will be understood. It seems to play such a central role in all the bizarre and specially interesting manifestations of the theory, from the nonlinearity of the equations to the occurrence of the operator $\ell^{\mu} \mathcal{H}_{\mu}$ in the secondary constraints of the canonical formalism.

It is quite possible, and even likely, that a major corollary of a successful development of a canonical formalism will be a clear cut definition of energy in the gravitational field. This is because a canonical formalism must of necessity define a quantity with the properties of energy, namely the operator which generates displacements in time. Professor Klein, who has laid great emphasis on the displacement properties of the energy operator in relativistic field theories, was impressed by the progress that DeWitt has achieved in the canonical approach, having been previously very pessimistic about the outlook for traditional methods. He was particularly pleased at DeWitt's demonstration of how easy it is to pass from the canonical formalism to the theory of Feynman and how the Dirac theory of constraints, which underlies DeWitt's work, contains Laurent's theorems on Feynman quantization as corollaries.

It is appropriate to conclude this report by repeating Klein's emphasis on the fact that the theory of gravitation is a theory of space and time. Whether this space-time has real nonlocal properties, what form these properties (if any) take, and whether the energy concept can be given a sharp definition, are still unknown, and it is impossible to say when the answers will be found. However, many new and interesting sides to these problems were learned and discovered by the participants at Copenhagen. This report has attempted to present the most important of these.

\section{References}

Dirac, P.A.M. 1938. Classical Theory of Radiating Electrons. Proc. Roy. Soc. A 167: $148-169$.

Dirac, P.A.M. 1950. Generalized Hamiltonian Dynamics. Can. J. Math. 2: 129-148.

11 The cross section still damps out at high frequencies. 
Klauder, J. and Wheeler, J.A. 1957. On the Question of a Neutrino Analog to Electric Charge. Rev. Mod. Phys. 29: 516-517.

Misner, C.W. 1957. Feynman Quantization of General Relativity. Rev. Mod. Phys. 29: 497-509.

Newton, T.D. and Wigner, E.P. 1949. Localized States for Elementary Systems. Rev. Mod. Phys. 21: 400-406.

Pauli, W. 1957. Lectures Delivered to the CERN Theoretical Study Division in 1956-1957.

Rainich, G.Y. 1925. Electrodynamics in the General Relativity Theory. Trans. Am. Math. Soc. 27: 106-136.

Weiss, P. 1938. On the Hamilton-Jacobi Theory and Quantization of a Dynamical Continuum. Proc. Roy. Soc. A 169: 102-119.

Open access funding provided by Max Planck Society.

Open Access This is an open access article distributed under the terms of the Creative Commons Attribution License (http://creativecommons.org/licenses/by/4.0), which permits unrestricted use, distribution, and reproduction in any medium, provided the original work is properly cited. 